\title{
Comparison of early postoperative results between conventional and transapical mitral valve repair
}

\author{
Aleksejus Zorinas ${ }^{1}$, Artūras Lipnevicius ${ }^{1}$, Viktorija Bleizgytèㄹ, Vilius Janušauskas ${ }^{1}$, Daina Liekienè ${ }^{1}$, \\ Mindaugas Budra ${ }^{1}$, Robertas Stasys Samalavičius ${ }^{2}$, Agnė Drąsutienè ${ }^{1}$, Diana Zakarkaitè ${ }^{1}$, Audrius Aidietis ${ }^{1}$, \\ Kęstutis Ručinskas ${ }^{1}$
}

${ }^{1}$ Clinic of Cardiac and Vascular Diseases, Vilnius University, Vilnius, Lithuania

${ }^{2}$ Centre of Anaesthesia, Intensive Care, and Pain Management, Department of Intensive Care, Vilnius University, Vilnius, Lithuania

Adv Interv Cardiol 2019; 15, 4 (58): 439-445

DOI: https://doi.org/10.5114/aic.2019.90218

\begin{abstract}
A bstract
Introduction: Conventional mitral valve repair (CMVR) is well-established, safe and effective treatment for degenerative mitral regurgitation (MR). Transapical off-pump implantation of artificial chordae (TA) has been introduced into practice and gained interest among surgeons. However, there are no publications comparing the results between TA and CMVR.

Aim: To compare early postoperative outcomes of CMVR with TA in patients with degenerative MR.

Material and methods: This was a retrospective cohort study. A total of 169 patients who underwent mitral valve repair between 2011 and 2018 were included in this analysis. Patients were divided into two groups: the TA group, $n=78$ and CMVR group, $n=91$. The groups were compared for early postoperative outcomes.

Results: Patients in the TA group were younger, $54.2 \pm 11.1$ vs. $59.5 \pm 12.8$ years $(p=0.005)$. Patients in the CMVR group had more complicated postoperative course with higher incidence of blood transfusion ( $42.9 \%$ vs. $7.8 \%, p=0.001)$, atrial fibrillation $(25.3 \%$ vs. $11.7 \%, p=0.031)$, renal insufficiency ( $15.4 \%$ vs. $2.6 \%, p=0.007)$ and stroke $(2.1 \%$ vs. $0 \%)$. In the early postoperative period, one patient died in the TA group, and there were no deaths in the CMVR group $(p=0.277)$. Residual moderate to severe mitral regurgitation was present in nine (11.5\%) TA patients, while none of the patients in the CMVR group had moderate or a higher degree of residual regurgitation $(p=0.001)$

Conclusions: Off-pump transapical MV repair is a feasible and safe procedure with low postoperative morbidity rates. Higher rates of mitral regurgitation reoccurrence would require a careful and thorough selection of the patients suitable for the TA approach.
\end{abstract}

Key words: minimally invasive surgery, mitral regurgitation, transapical mitral valve repair.

S u m m a ry

Degenerative mitral valve disease is a common cause of primary mitral regurgitation. Conventional mitral repair is an established treatment for this pathology. The development of less invasive conventional mitral repair approaches is aimed to minimize surgical trauma, thus enhancing recovery. However, patients are still exposed to risk related to the use of cardiopulmonary bypass and aortic cross-clamp. Recently presented techniques of transapical mitral repair allow the implantation of artificial chordae to the prolapsing or flailing mitral leaflet without exposure to the risk factors mentioned above. Transapical implantation of artificial chordae results in immediate physiological restoration of the mitral valve anatomy intraoperatively while respecting the dynamic shape of the mitral apparatus. Although single centers have published their experience of this treatment modality, there have been no trials comparing outcomes of conventional and transapical mitral repair. Taking into the account early results of our retrospective analysis, we believe that transapical mitral repair is a feasible and safe procedure. Transapical mitral repair demonstrated good early postoperative results with low postoperative blood loss and a low rate of blood product transfusion. However, compared to the conventional mitral repair, it has a greater recurrence rate of moderate or severe mitral regurgitation. Therefore, careful selection of eligible patients for transapical mitral repair by a highly experienced team is imperative.

\section{Corresponding author:}

Aleksejus Zorinas PhD, Clinic of Cardiac and Vascular Diseases, Vilnius University, 2 Santariskiu St, 04816 Vilnius, Lithuania, phone: +37 069829079, e-mail: aleksejus.zorinas@santa.lt

Received: 17.06.2019, accepted: 4.09.2019. 


\section{Introduction}

Degenerative mitral valve (MV) disease is a common cause of primary mitral regurgitation (MR) in developed countries [1]. Reconstructive MV surgery has become an established gold standard treatment for this condition. Development of less invasive surgical approaches is aimed at minimizing surgical trauma and enhancing recovery; however, the patients still remain exposed to risks of cardiopulmonary bypass (CPB) and aortic crossclamp.

Recently developed techniques of transapical MV repair allow the implantation of artificial expanded polytetrafluoroethylene (ePTFE) chordae to the prolapsing or flailing mitral leaflet without CPB. These techniques are truly minimally invasive as only a small incision of the chest at the apex of the left ventricle (LV) is required to access the MV.

Transapical MV repair results in immediate physiological restoration of the $M V$ anatomy intraoperatively while respecting the dynamic shape of the $\operatorname{MV}[2,3]$.

Although individual centers have published their experience of transapical mitral repair, there have been no trials where early outcomes of conventional and transapical MV repair are compared.

This is a retrospective non-randomized study with a patient cohort at a single center.

\section{Aim}

The aim of the study was to compare early postoperative results following conventional MV repair (CMVR) and transapical off-pump mitral repair (TA) using the NeoChord DS1000 device (NeoChord Inc., Minneapolis, MN, USA).

\section{Material and methods}

The study involved 169 patients who underwent MV repair for severe degenerative MV disease between 2011 and 2018. Transthoracic echocardiography and 2D/3D transesophageal echocardiography were used for selection of the patients in both treatment groups. All candidates had indications for surgical MV repair according to the current ACC/AHA and ESC/EACTS guidelines $[4,5]$. This study was approved by the local Regional Bioethics Committee.

MV pathology included single or bi-leaflet MV prolapse or flail, with or without chordal rupture. Patients with a restrictive mechanism of regurgitation (rheumatic disease, cardiomyopathy), ischemic mitral regurgitation, $M V$ infectious lesions and patients with a central regurgitation jet were excluded from the study. All patients with degenerative MV disease were discussed by the Heart Team for eligibility to perform either CMVR surgery or a TA procedure. Patients with a favorable MV anatomy who agreed to undergo a transapical procedure were selected for the TA.
Patients were divided into two groups according to the type of surgery: the TA group and the CMVR group.

Seventy-eight patients underwent a TA procedure. Flailing leaflet with rupture of one or more chordae was revealed in $60(77 \%)$ patients. The rest of the patients had MR due to a prolapsing leaflet with intact native chordae. Patients in the TA group were stratified into 4 categories as per anatomical type of mitral pathology (2): type A (15 patients), isolated P2 prolapse; type B (43 patients), disease of the P2 and adjacent segments; type C (16 patients), single or bi-leaflet prolapse with peri-commissural segments involved; type D (4 patients), isolated A2 prolapse.

The CMVR group included 91 patients who underwent on-pump conventional MV repair. The patients were also stratified according to the type of mitral pathology: type A, 11 patients; type B, 18 patients; type C, 60 patients; type D, 2 patients.

Patients in both groups underwent MV repair during the same time frame. Type B patients mostly underwent transapical mitral repair, while type $C$ patients were mostly treated with a conventional surgical procedure.

\section{Statistical analysis}

Statistical analysis was performed using the data acquisition and analysis software package SPSS 21.0 (IBM Corp., Armonk, NY, USA). The quantitative normality of continuous data was evaluated using the criteria of histograms, rectangular diagrams, and the Shapiro-Wilk test $(p>0.05)$. Quantitative data with a normal distribution are presented as a mean value \pm standard deviation. Student's $t$ test for independent samples was used to compare the mean values. The quantitative continuous data outside the normal distribution are presented as median and quartile intervals. The Mann-Whitney-Wilcoxon test was used to compare such data. The categorical data are expressed as a percentage. Their variables were compared using the $\chi^{2}$ or Fisher criterion. A $p$-value less than 0.05 was regarded as statistically significant.

\section{Results}

Demographics and comorbidities

Patients in the TA group were older, $59.5 \pm 12.8$ vs. $54.2 \pm 11.1$ years $(p=0.005)$. Male gender was dominant in both groups (Table I).

STS and EuroSCORE II risk scores did not differ between the groups: $0.47 \%$ and $0.83 \%$ in the TA group vs. $0.43 \%$ and $0.84 \%$ in the CMVR group, respectively.

Patients in the CMVR group were of higher NYHA functional class: $74.7 \%$ presented with NYHA III class vs. $35.9 \%$ in the TA group. The patients in the CMVR group had a higher incidence of both paroxysmal (22\% vs. $6.4 \%)$ and permanent (17.6\% vs. $0 \%$ ) atrial fibrillation preoperatively. 
Table I. Demographics and comorbidities

\begin{tabular}{|c|c|c|c|}
\hline Variables & CMVR group & TA group & $P$-value \\
\hline Patients, $n$ & 91 & 78 & \\
\hline Male patients, $n(\%)$ & $52(57.1)$ & $53(67.9)$ & 0.099 \\
\hline Age, mean \pm SD [years] & $54.2 \pm 11.1$ & $59.5 \pm 12.8$ & 0.005 \\
\hline $\mathrm{BMI}$, mean $\pm \mathrm{SD}\left[\mathrm{kg} / \mathrm{m}^{2}\right]$ & $26.4 \pm 6.1$ & $26.8 \pm 4.6$ & 0.634 \\
\hline STS score (\%), median (IQR) & $0.43(0.31-0.70)$ & $0.47(0.24-0.74)$ & 0.142 \\
\hline EuroSCORE II (\%), median (IQR) & $0.84(0.67-1.13)$ & $0.83(0.67-1.35)$ & 0.689 \\
\hline NYHA I, $n(\%)$ & $1(1.1)$ & $4(5.1)$ & 0.123 \\
\hline NYHA II, $n(\%)$ & $21(23.1)$ & $45(57.7)$ & 0.001 \\
\hline NYHA III, $n(\%)$ & $68(74.7)$ & $28(35.9)$ & 0.001 \\
\hline NYHA IV, $n(\%)$ & $1(1.1)$ & $1(1.3)$ & 0.912 \\
\hline Creatinine, median $(\mathrm{IQR})[\mu \mathrm{mol} / \mathrm{I}]$ & $78(68-93)$ & $74(64-92)$ & 0.220 \\
\hline Creatinine clearance, mean $\pm \mathrm{SD}[\mathrm{ml} / \mathrm{min}]$ & $100 \pm 31.9$ & $99.6 \pm 34.6$ & 0.987 \\
\hline Creatinine clearance less than $85 \mathrm{ml} / \mathrm{min}, n(\%)$ & $24(26.37)$ & $24(31.2)$ & 0.525 \\
\hline CAD, $n(\%)$ & $5(5.5)$ & $6(7.7)$ & 0.394 \\
\hline Diabetes, $n(\%)$ & $5(5.5)$ & $2(2.6)$ & 0.290 \\
\hline COPD, $n(\%)$ & $1(1.1)$ & $3(3.8)$ & 0.11 \\
\hline Preoperative stroke, $n(\%)$ & $2(2.2)$ & $3(3.8)$ & 0.427 \\
\hline Pulmonary hypertension (> $55 \mathrm{~mm} \mathrm{Hg}$ ), $n(\%)$ & $13(14.29)$ & $10(12.8)$ & 0.208 \\
\hline Paroxysmal AF, $n(\%)$ & $20(22)$ & $5(6.4)$ & 0.004 \\
\hline Permanent $\mathrm{AF}, n(\%)$ & $16(17.6)$ & 0 & 0.000 \\
\hline Pacemaker, $n(\%)$ & $1(1.1)$ & 0 & 0.538 \\
\hline Hypertension, $n(\%)$ & $36(39.6)$ & $42(53.8)$ & 0.044 \\
\hline
\end{tabular}

$A F$ - atrial fibrillation, $B M I$ - body mass index, CAD - coronary artery disease, CMVR - conventional mitral valve repair, COPD - chronic obstructive pulmonary disease, EuroSCORE - European System for Cardiac Operative Risk Evaluation, IQR - interquartile range, NYHA - New York Heart Association, SD - standard deviation, STS - Society of Thoracic Surgeons, TA - transapical.

\section{Preoperative echocardiographic data}

There was no significant difference in left ventricular systolic function between the groups (Table II). Echocardiography revealed a slightly increased left atrial volume and mitral annular dimensions in the CMVR group.

All patients in both groups underwent surgery for severe mitral regurgitation. There was a higher incidence of significant tricuspid regurgitation (TR) in the CMVR group: $35.2 \%$ of CMVR patients had moderate TR vs. $14.1 \%$ in the TA group ( $p=0.002)$.

\section{Intraoperative data and early postoperative results}

In the CMVR group, 75 (82.4\%) procedures were performed using artificial ePTFE chordae; an annuloplasty ring was used in 89 (97.8\%) cases, and concomitant TV procedures were performed in 79 (86.8\%) patients
(TV Kay bicuspidalization in 74 cases and ring annuloplasty in 5 cases).

The median duration of surgery among CMVR patients was longer as compared to the TA procedure -312 min vs. 120 min (Table III).

There was one conversion to conventional repair in the TA group, mitral repair failed as a result of iatrogenic injury to the posterior MV leaflet caused by the artificial chord delivery device.

The average number of implanted ePTFE chordae in the TA group was 3.5 per patient (range: 1-7 per patient). Three or four chordae were implanted in 52 (68\%) patients. The most common site for the implantation was the P2 segment (92\% patients). A good intraoperative result with no residual MR was achieved in 43 (56\%) and mild MR in 28 (36\%) patients. There were 4 (5\%) patients with residual moderate MR and 2 (3\%) patients with failed repair and residual severe MR. They did not 
Table II. Preoperative echocardiographic data

\begin{tabular}{|c|c|c|c|}
\hline Parameter & CMVR group & TA group & $P$-value \\
\hline $\operatorname{LVEF}(\%)$, mean \pm SD & $56 \pm 6$ & $57 \pm 5$ & 0.401 \\
\hline LVEDD, mean $\pm \mathrm{SD}[\mathrm{mm}]$ & $59.8 \pm 7$ & $59.1 \pm 6.2$ & 0.540 \\
\hline LVESD, mean \pm SD $[\mathrm{mm}]$ & $36.5 \pm 7.5$ & $35.6 \pm 6.3$ & 0.429 \\
\hline $\mathrm{LA}$ volume index, mean $\pm \mathrm{SD}\left[\mathrm{ml} / \mathrm{m}^{2}\right]$ & $75 \pm 24$ & $73 \pm 26$ & 0.596 \\
\hline MV annulus AP diameter, mean $\pm \mathrm{SD}[\mathrm{mm}]$ & $38.2 \pm 6.6$ & $36.2 \pm 5.6$ & 0.064 \\
\hline MV annulus ML diameter, mean \pm SD [mm] & $46.6 \pm 6.5$ & $44.7 \pm 6.5$ & 0.100 \\
\hline MR severe, $n(\%)$ & $91(100)$ & $78(100)$ & 0.330 \\
\hline TR none/trivial, $n$ (\%) & $27(29.6)$ & $34(43.6)$ & 0.060 \\
\hline TR mild, $n(\%)$ & $32(35.2)$ & $33(42.3)$ & 0.341 \\
\hline TR moderate, $n(\%)$ & $32(35.2)$ & $11(14.1)$ & 0.002 \\
\hline
\end{tabular}

$A P$ - antero-posterior, CMVR - conventional mitral valve repair, $L A$ - left atrium, LVEDD - left ventricular end-diastolic diameter, $L V E F$ - left ventricular ejection fraction, LVESD - left ventricular end-systolic diameter, $M L$ - medio-lateral, MR - mitral regurgitation, $M V$ - mitral valve, SD - standard deviation, TA - transapical, TR - tricuspid regurgitation.

Table III. Intraoperative data and early postoperative results

\begin{tabular}{|c|c|c|c|}
\hline Variables & CMVR group & TA group & $P$-value \\
\hline \multicolumn{4}{|l|}{ Intraoperative variables: } \\
\hline Duration of surgery, median (IQR) [min] & $312(280-361)$ & $120(110-146)$ & $<0.001$ \\
\hline CPB, median (IQR) [min] & $178(149-206)$ & NA & \\
\hline Aortic cross-clamp time, median (IQR) [min] & $123(96-149)$ & NA & \\
\hline TV repair & $79(86.8)$ & NA & \\
\hline Conversion to full sternotomy, $n(\%)$ & NA & $1(1.3)$ & \\
\hline Residual MR none/trivial, $n$ (\%) & $75(82.4)$ & $43(55.8)$ & $<0.001$ \\
\hline Residual MR mild, $n(\%)$ & $16(17.6)$ & $28(36.4)$ & 0.007 \\
\hline Residual MR moderate, $n$ (\%) & 0 & $4(5.2)$ & 0.03 \\
\hline Residual MR severe, $n(\%)$ & 0 & $2(2.6)$ & 0.12 \\
\hline \multicolumn{4}{|l|}{ Blood products: } \\
\hline Transfusion of RBC, $n(\%)$ & $39(42.9)$ & $6(7.8)$ & $<0.001$ \\
\hline Transfusion of platelets, $n(\%)$ & $9(9.9)$ & $2(2.6)$ & 0.057 \\
\hline Transfusion of FFP, $n(\%)$ & $17(18.7)$ & $3(3.9)$ & 0.03 \\
\hline Blood products (total), $n(\%)$ & $43(47.3)$ & $6(7.8)$ & $<0.001$ \\
\hline \multicolumn{4}{|l|}{ Postoperative variables: } \\
\hline Chest tube drainage, median (IQR) [ml] & $300(200-550)$ & $200(150-300)$ & 0.001 \\
\hline New atrial fibrillation, $n(\%)$ & $23(25.3)$ & $9(11.7)$ & 0.031 \\
\hline New PPM within 30 days postoperatively, $n$ (\%) & $11(12.1)$ & $2(2.6)$ & 0.003 \\
\hline Re-exploration, $n(\%)$ & $3(3.3)$ & $3(3.9)$ & 0.577 \\
\hline Duration of postoperative CMV, median (IQR) [h] & $7(514)$ & $4(2.5-5)$ & $<0.001$ \\
\hline Length of ICU stay, median (IQR) [h] & $67.5(44-113)$ & $22(20-24)$ & $<0.001$ \\
\hline Myocardial infarction, $n(\%)$ & 0 & 0 & \\
\hline Stroke, $n(\%)$ & $2(2.2)$ & 0 & 0.191 \\
\hline Wound infection, $n(\%)$ & $1(1.1)$ & 0 & 0.354 \\
\hline Renal failure (creatinine elevation by $150 \%), n(\%)$ & $14(15.4)$ & $2(2.6)$ & 0.007 \\
\hline Hemofiltration, $n(\%)$ & $3(3.3)$ & 0 & 0.106 \\
\hline Hospital stay, median (IQR) [days] & $16(14-21)$ & $8(7-9)$ & $<0.001$ \\
\hline Mortality, $n(\%)$ & 0 & $1(1.3)$ & 0.277 \\
\hline MR severe at 30 days & 0 & $9(11.7)$ & 0.001 \\
\hline
\end{tabular}

$C M V$ - controlled mechanical ventilation, CMVR - conventional mitral valve repair, CPB - cardiopulmonary bypass, FFP - fresh frozen plasma, ICU - intensive care unit, IQR - interquartile range, $M R$ - mitral regurgitation, PPM - permanent pacemaker, $R B C$ - red blood cells, TA - transapical, TV - tricuspid valve. 
undergo any further procedure as they were reluctant to undergo conventional repair at the time of consent.

In the CMVR group, the median number of implanted PTFE chordae was 2 per patient (IQR: 2-3). No residual MR was achieved in 75 (82.4\%) patients and mild MR in 16 (17.6\%). A second pump run due to an unacceptable result of the repair was necessary in $3(3.3 \%)$ patients.

At 30 days, none of the patients in the CMVR group had severe MR, while in the TA group, $9(11.7 \%, p=0.001)$ patients had moderate or severe MR (2 (2.6\%) patients had moderate and 7 (9.1\%) severe).

Postoperative blood loss in the TA group was significantly lower compared to the CMVR group, $200 \mathrm{ml}$ vs. $300 \mathrm{ml}, p=0.001$. It determined a significantly lower rate of blood product transfusion in the TA group of patients (7.8\% vs. $42.9 \%$ for RBC, $p<0.005 ; 7.8 \%$ vs. $47.3 \%$ for all blood products, $p<0.001)$. There was no difference in re-exploration rate between the groups.

One patient died in the TA group on the $2^{\text {nd }}$ postoperative day. Death occurred due to bleeding-related cardiac tamponade. Following evacuation of the pericardial tamponade, the patient immediately developed ventricular fibrillation. Direct cardiac compressions led to rupture of the right ventricle with a subsequent lethal outcome.

Early postoperative mortality was $1.3 \%$ in the TA group and $0 \%$ in the CMVR group.

Patients in the CMVR group had a higher rate of postoperative atrial fibrillation $(11.7 \%$ vs. $25.3 \%, p<0.05)$ and PPM insertion (2.6\% vs. $12.1 \%, p<0.05)$. After the TA procedure, a permanent pacemaker was implanted in two patients; those were patients with preoperatively known sick sinus node syndrome.

Median time to weaning from ventilation was significantly shorter in the TA group: $4 \mathrm{~h}$ vs. $7 \mathrm{~h}(p<0.005)$. There was a substantial difference in the ICU length of stay between the TA and CMVR groups ( $22 \mathrm{~h}$ vs. $67.5 \mathrm{~h}$, $p<0.005)$ as well as postoperative in-hospital stay (8 days vs. 16 days, $p<0.005$ ).

The rate of postoperative stroke was $2.2 \%$ in CMVR patients, and there was one patient with postoperative wound infection (1.1\%). None of these complications were recorded in the TA group.

Postoperative renal failure (elevation of creatinine level $>150 \%$ above baseline) was more common in the CMVR group (15.4\% vs. $2.6 \%$ ). Three (3.3\%) patients in the CMVR group and none in the TA group required hemofiltration or hemodialysis.

\section{Discussion}

The ePTFE chord has become a standard material in modern MV surgery, and recent studies have shown reliable long-term results of mitral repair using ePTFE chordae $[6,7]$. Castillo et al. reported the absence of moderate or severe MR to be $90.3 \pm 3.7 \%$ at 7 years [6]. The David group published 25-year results showing freedom from reoperation of $90.2 \%$ and freedom from recurrent severe MR of $91.0 \%$ after 18 years [7].

The recent concept of transapical delivery of ePTFE chords was developed in order to reduce the invasiveness of the surgery and the rate of complications related to the use of cardiopulmonary bypass. This type of repair also enhances postoperative recovery of the patient and reduces duration of ICU and in-hospital stay [8].

Since the introduction of the technology, more than 1000 patients in Europe and the USA have successfully undergone the NeoChord procedure [9]. This system allows minimally invasive off-pump delivery of the ePTFE chordae to the prolapsing or flail MV leaflet and enables the adjustment of the length of the chordae under direct echocardiographic control while the valve is fully functioning under physiologic conditions of the beating heart. This TEE guided procedure is currently standardized and is described in detail in recent publications of Colli et al. [5, 9] and Samalavicius et al. [10].

Another novel technology for transapical mitral repair is the Harpoon Mitral Valve Repair System (H-MVRS; Harpoon Medical Inc., Baltimore, Maryland, USA). It uses a similar approach to the NeoChord system; however, the mechanism of ePTFE chordae anchoring is different: the device perforates the leaflet and anchors the artificial chord by a self-tying suture knot [1, 11]. According to 2019 data, there were 65 patients who underwent this procedure, and it was successful in 62 (95.4\%) patients. There was no early mortality or major adverse events. The reported rate of AF was $18 \%$. However, $14 \%$ of the patients had reoperation and another $2 \%$ had severe residual MR at 12 months [12].

Multiple reports have demonstrated the safety and feasibility of transapical MV repair using the NeoChord DS1000 device with an effective reduction of MR achieved during the procedure $[8,13]$. Colli et al. recently published procedural outcomes and 12-month follow-up data of the largest series at a single institution [14], followed by a manuscript presenting multicenter European experience [8]. Reported intraoperative results are quite similar to those presented in this study: two thirds of the patients received three or four artificial chordae, rates of conversion to conventional surgery and mortality were $1.4-1.9 \%$ vs. $1.3 \%$ in this series. Procedural success was slightly lower in our series - MR reduction to mild or less was achieved in 71 (91\%) patients compared to 96.7$98.6 \%$ reported by Colli et al. The fact that there were more patients with no residual MR in the CMVR group could be explained by a wider choice of components in the conventional repair such as annuloplasty ring, closure of clefts and others. Furthermore, patients who had higher than mild residual MR, which could not be repaired any further, underwent $M V$ replacement and were not included in this study.

Our experience shows that the NeoChord procedure is beneficial only for patients with a non-complicated 
lesion of the mitral valve (mostly isolated P2 prolapse or flail, type A and B) at the early stages of the disease when the valve can be repaired by isolated implantation of the artificial chordae. Other patients with more complex mitral pathology, multiple prolapsing segments, annular dilatation, poor predicted coaptation and paracommissural disease (type C) should undergo conventional surgery. Conventional surgery can offer more options for a better result, and also chances of unsuccessful repair and necessity for replacement of the valve in these patients are higher. The subgroup of patients with a more complex MR anatomy (type C) was larger in the CMVR group, due to selection bias of the investigators, meaning that patients with more favorable anatomy (i.e. types $A$ and $B$ ) were selected for the TA repair.

Modern direct or indirect transcatheter annuloplasty devices allow transapical repair to be used in more complex patients. Six recently reported cases of transapical mitral repair and concomitant annuloplasty using annuloplasty transcatheter devices (COMBO approach) showed one of the potential directions for further development of transapical technology [15]. Addition of an annuloplasty option to transapical repair would improve the results of transapical repair, particularly in complex mitral pathology with annular dilatation.

Although the conventional approach can often be beneficial for patients with complex mitral pathology, our study shows a lower rate of postoperative adverse events in the TA group compared with the CMVR group. Transapical repair is a much less invasive procedure; therefore, both postoperative bleeding and rates of blood products are much lower. Red blood cell transfusion was required in $6(7.8 \%)$ patients and this is very close to the $8 \%$ rate reported by Colli et al. in the multicenter European study. Meanwhile the most commonly reported incidence is about $50 \%$ after conventional and about $40 \%$ after minimally invasive mitral surgery [16]. Even though transfusions were rare in the TA group, the incidence of re-exploration was similar in both groups (3.3\% vs. 3.9\%) and higher in comparison to European experience (1.4\%).

Postoperative atrial fibrillation affects up to a third of patients after conventional surgery and $15-20 \%$ of patients following minimally invasive mitral surgery [16-18]. Our study shows significant reduction in AF rate after a transapical procedure (11.7\% vs. $25.3 \%)$, although multicenter NeoChord data report the incidence to be 22.5\% [8].

Postoperative neurologic complications are often related to CPB and cross-clamping of the aorta with a reported range of $0.7-2.8 \%[18,19]$. Both our study and European experience show that the transapical approach helps to avoid this complication [8].

Significantly shorter postoperative recovery in comparison to conventional surgery, including shorter mechanical ventilation, ICU stay, and postoperative in-hospi- tal stay, is often emphasized in the publications reporting outcomes of transapical repair. In this study the duration of ventilation and in-hospital stay in the CMVR group was almost double compared to the TA group.

Our study has demonstrated safety and feasibility of the TA procedure with a lower rate of postoperative adverse events in the TA group in comparison to conventional surgery. Nevertheless, the TA procedure is associated with a higher rate of early postoperative failures: no patients in the CMVR group developed severe MR at 30 days; meanwhile, severe residual MR was present in $2(2.6 \%)$ patients immediately after the procedure and in $7(9.1 \%)$ patients at 30-day follow-up.

Both patients with severe intraprocedural residual $M R$ and 5 out of 7 patients with severe MR at 30-day follow-up were type $C$ as per anatomical changes preoperatively. Therefore, we conclude that type $C$ patients should not be considered as candidates for a transapical procedure due to high recurrence of MR.

\section{Study strengths and limitations}

This is a retrospective non-randomized analysis with a limited number of patients. The Heart Team biased the patient selection for the procedure, according to MV anatomy. The patients in the TA and CMVR groups were not equal; there were more patients with complex MV pathology (group C) in the CMVR group. The groups were too small to perform propensity matching.

\section{Conclusions}

Transapical MV repair using the NeoChord DS1000 device is a feasible and relatively safe procedure. The procedure time is relatively short and MV repair is performed without exposing the patient to CPB-related risks. Mitral repair using the NeoChord device demonstrated good early postoperative results with low postoperative blood loss and a low rate of blood product transfusion. However, transapical MV repair using the NeoChord DS1000 device, as compared to conventional repair, has a greater recurrence rate of severe MR. Therefore, careful selection of eligible patients by a highly experienced team is imperative.

\section{Conflict of interest}

Dr. A. Drąsutienè, D. Zakarkaitè and A. Aidietis have worked as consultants for NeoChord Inc. The others authors declare no conflict of interest.

\section{References}

1. Lancellotti P, Radermecker M, Durieux R, et al. Transapical beating-heart chordae implantation in mitral regurgitation: a new horizon for repairing mitral valve prolapse. J Thorac Dis 2016; 8: E1665-71.

2. Colli A, Besola L, Montagner M, et al. Acute intraoperative echocardiographic changes after transapical off-pump mitral valve 
repair with NeoChord implantation. Int J Cardiol 2018; 257: 230-4.

3. Colli A, Bizzotto E, Pittarello D, Gerosa G. Beating heart mitral valve repair with neochordae implantation: real-time monitoring of haemodynamic recovery. Eur J Cardiothorac Surg 2017; 52: 991-2.

4. Falk V, Baumgartner H, Bax JJ, et al. Corrigendum to '2017 ESC/ EACTS Guidelines for the management of valvular heart disease' [Eur J Cardiothorac Surg 2017; 52: 616-664]. Eur J Cardiothorac Surg 2017; 52: 832.

5. Nishimura RA, Otto CM, Bonow RO, et al. 2017 AHA/ACC Focused Update of the 2014 AHA/ACC Guideline for the Management of Patients With Valvular Heart Disease: A Report of the American College of Cardiology/American Heart Association Task Force on Clinical Practice Guidelines. J Am Coll Cardiol 2017; 70: 252-89.

6. Castillo JG, Anyanwu AC, El-Eshmawi A, Adams DH. All anterior and bileaflet mitral valve prolapses are repairable in the modern era of reconstructive surgery. Eur J Cardiothorac Surg 2014; 45: 139-45.

7. David TE, Armstrong S, Ivanov J. Chordal replacement with polytetrafluoroethylene sutures for mitral valve repair: a 25 -year experience. J Thorac Cardiovasc Surg 2013; 145: 1563-9.

8. Colli A, Manzan E, Aidietis A, et al. An early European experience with transapical off-pump mitral valve repair with NeoChord implantation. Eur J Cardiothorac Surg 2018; 54: 460-6.

9. Colli A, Adams D, Fiocco A, et al. Transapical NeoChord mitral valve repair. Ann Cardiothorac Surg 2018; 7: 812-20.

10. Samalavicius RS, Norkiene I, Drasutiene A, et al. Anesthetic management and procedural outcomes of patients undergoing off-pump transapical implantation of artificial chordae to correct mitral regurgitation: case series of 76 patients. Anesth Analg 2018; 126: 776-84.

11. Kiefer P, Meier S, Noack T, et al. Good 5-year durability of transapical beating heart off-pump mitral valve repair with neochordae. Ann Thorac Surg 2018; 106: 440-5.

12. Gammie J. Harpoon: Technology and Clinical Updates. TVT 2019; Chicago, IL, 12-15 June 2019.

13. Colli A, Manzan E, Zucchetta F, et al. Feasibility of anterior mitral leaflet flail repair with transapical beating-heart neochord implantation. JACC Cardiovasc Interv 2014; 7: 1320-1.

14. Colli A, Manzan E, Besola L, et al. One-year outcomes after transapical echocardiography-guided mitral valve repair. Circulation 2018; 138: 843-5.

15. Colli A, Beiras-Fernandez A, Ruf T, et al. Transcatheter mitral valve repair: single stage combo approach. Rev Esp Cardiol 2019; 72: $972-5$

16. Gammie JS, Zhao Y, Peterson ED, et al. J. Maxwell Chamberlain Memorial Paper for adult cardiac surgery. Less-invasive mitral valve operations: trends and outcomes from the Society of Thoracic Surgeons Adult Cardiac Surgery Database. Ann Thorac Surg 2010; 90: 1401-8, 10 e1; discussion 8-10.

17. Glauber M, Miceli A, Canarutto D, et al. Early and long-term outcomes of minimally invasive mitral valve surgery through right minithoracotomy: a 10-year experience in 1604 patients. J Cardiothorac Surg 2015; 10: 181.

18. Goldstone AB, Atluri P, Szeto WY, et al. Minimally invasive approach provides at least equivalent results for surgical correction of mitral regurgitation: a propensity-matched comparison. J Thorac Cardiovasc Surg 2013; 145: 748-56.

19. Speziale G, Nasso G, Esposito G, et al. Results of mitral valve repair for Barlow disease (bileaflet prolapse) via right minithora- cotomy versus conventional median sternotomy: a randomized trial. J Thorac Cardiovasc Surg 2011; 142: 77-83. 